The AstrophysiCAL JourNAL, 397:L107-L110, 1992 October 1

(C) 1992. The American Astronomical Society. All rights reserved. Printed in U.S.A.

\title{
RESOLVING THE FORBIDDEN-LINE EMISSION REGIONS OF YOUNG STELLAR OBJECTS: DD TAURI
}

\author{
Ana I. Gomez de Castro ${ }^{1,2,3}$ AND Ralph E. Pudritz ${ }^{1,3}$ \\ Received 1991 August 27; accepted 1992 July 14
}

\begin{abstract}
We present high spatial resolution, narrow-band images of the T Tauri star, DD Tau, obtained with the High-Resolution Camera of CFHT. We have resolved the forbidden line emission region (FLER) of this source, on a spatial scale 0.5 in both [N II] and [S II]. The bulk of the continuum emission is concentrated in two knots that are separated by 0.55 (77 AU) and oriented in the direction P.A. $=6.4$. The forbidden-line morphology is distinctly different from that of the continuum. The [N II] emission appears to be a "jetlike" extension connecting the two continuum knots. The [S II] emission seems to be confined to the northern knot. The southern knot (DD Tau B) is extended in the direction P.A. $\simeq 130^{\circ}$. The orientation of the disk as defined by IR polarization measurements is P.A. $\simeq 125^{\circ}$. We compare our observations with the existing theoretical models for the origin of FLERs.
\end{abstract}

Subject headings: ISM : general - line: profiles - stars: formation - stars: individual: DD Tauri stars: pre-main-sequence

\section{INTRODUCTION}

Forbidden-line emission regions are often associated with young stellar objects (YSOs) (Appenzeller \& Mundt 1989; Edwards et al. 1987). Most of these [O I], [S II], and [N II] emitting regions are unresolved at $1^{\prime \prime}$ (140 AU at the distance of Taurus). The equivalent widths of the forbidden lines are strongly correlated with the infrared excess of YSOs suggesting that underlying accretion disks, suspected to exist in many of these systems, are an essential ingredient in any model (e.g., Cabrit et al. 1990). Some of the YSOs with FLERs are also associated with optical jets. Long-slit, high spatial resolution spectra of optical jets show that their electron density increases smoothly toward the YSO up to values characteristic of the FLERs (Mundt et al. 1990). This suggests that there is a connection between FLERs and jets.

Standard stellar wind models fail to produce FLERs because radiative losses and adiabatic expansion cool the wind to below $1000 \mathrm{~K}$ within $0.2 \mathrm{AU}$ of the YSO, where densities are still much too high for the formation of lines such as [S II]. An effective means of heating gas far from T Tauri stars (TTS) is by shocks (Hartmann \& Raymond 1988). Since forbidden lines serve as highly specific shock diagnostics, FLERs constitute ideal probes of outflows on scales within 70 AU of the YSO.

Given that current models (e.g., Hartmann \& Raymond 1988; Bouvier et al. 1992; Gomez de Castro \& Pudritz 1992, hereafter GP) make different predictions about the origin of FLERs, we obtained high-resolution (typically, FWHM =0".5) images (using HR Cam at CFHT) of the FLER source, DD Tau. We discuss the observations in $\S 2$, analyze our results in $\S 3$, and discuss their implications in $\S 4$.

\footnotetext{
${ }^{1}$ Department of Physics and Astronomy, McMaster University, Hamilton, Ontario, Canada L8S 4M1 (postal address for R. E. P.)

${ }^{2}$ IUE Observatory-VILSPA, P.O. Box 50727, 28080 Madrid, Spain (postal address for A. I. G. de C.)

${ }^{3}$ Visiting Astronomer, Canada-France-Hawaii Telescope, operated by the National Research Council of Canada, the Centre National de La Recherche Scientifique de France, and the University of Hawaii.
}

\section{OBSERVATIONS}

DD Tau is the only TTS having both a red- and a blueshifted component of the forbidden lines, equally displaced in velocity with respect to the rest wavelength (see, e.g., Edwards et al. 1989). This fact strongly suggests that the system is nearly edge-on and that therefore, DD Tau ought to be the best candidate for resolving the FLER.

The observations were carried out in 1991 February with the High-Resolution Camera (HR Cam) of the Canada-FranceHawaii Telescope (CFHT). This camera enabled us to obtain images with spatial resolution of 0 ."5-0"8 (70-112 AU at Taurus). We employed the SAIC 2 CCD which has a field of view of $2.2 \times 2.2$ and a pixel size of $18 \mu \mathrm{m}$. The scale is 0.13 pixel $^{-1}$. We used the only other bright star in the field, $\mathrm{CZ}$ Tau, as the guide star (see McClure et al. 1989 for details on HR Cam).

We obtained images in three narrow-band filters with the specifications [O $\mathrm{I}] \lambda 5577\left(\lambda_{c}=5581 \AA\right.$ and FWHM $\left.=10 \AA\right)$, [S II] $\lambda 6729\left(\lambda_{c}=6729 \AA\right.$ and FWHM $\left.=47 \AA\right)$, and [N II] $\lambda 6584\left(\lambda_{c}=6584 \AA\right.$ and FWHM $\left.=17 \AA\right)$, where $\lambda_{c}$ is the line center. We also used an intermediate-band filter centered at $6100 \AA\left(\lambda_{c}=6100 \AA\right.$ and FWHM $\left.=198 \AA\right)$ in order to study the continuum at wavelengths comparable to those of the narrow-band filters. The filter integrals $\left[\int T(\lambda) \delta \lambda\right.$ where $T(\lambda)$ represents the filter transmittance] are $4.7 \AA, 10.8 \AA, 29.0 \AA$, and $142.1 \AA$ for the $[\mathrm{O} \mathrm{I}],[\mathrm{N} \mathrm{II}],[\mathrm{S}$ II] and continuum filters, respectively. We obtained four images in each bandpass for a total exposure time of $1 \mathrm{hr}$ in the narrow-band filters and a total of 15 minutes for the continuum. The data were reduced using the IRAF and MIDAS image processing packages. Images were bias-corrected and flat-fielded in the standard manner.

\section{RESULTS}

Contour plots of the best images (as oriented on the CCD: $x$ and $y$ axes are parallel to rows and columns, respectively) taken with each filter appear in Figure 1. The images show that DD Tau is dominated by a northern (A) and a southern (B) 

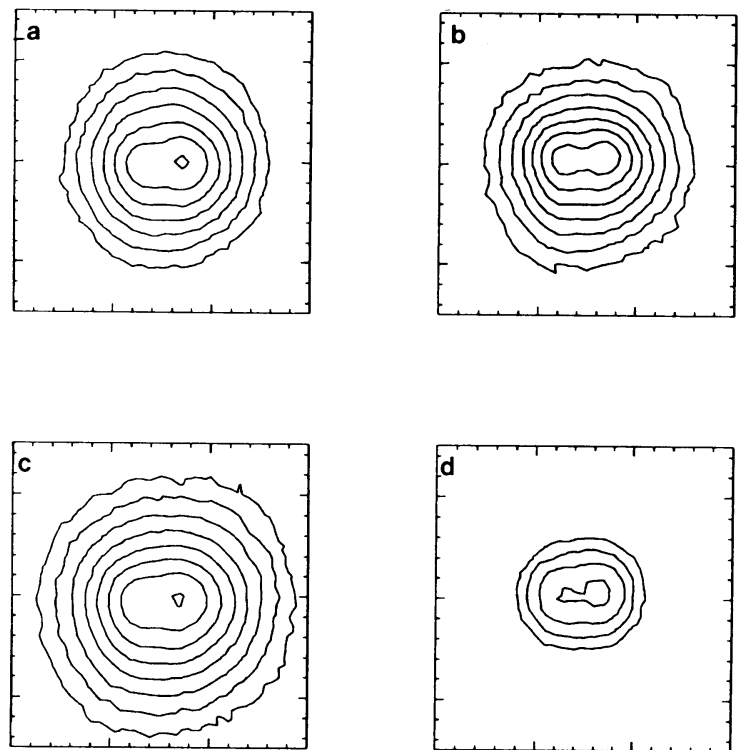

FIG. 1.-Contour plots of our best images of DD Tau. Identical levels are plotted in each frame and correspond to $16,32,64,128,256,512,1024,2048$, and $4096 \sigma$. The horizontal ( $x$-axis) makes an angle of 9.1 with the iorth North is at the left, and east at the top. $(a)[\mathrm{N} \mathrm{II}]-2 ;(b)[\mathrm{S} \mathrm{II}]-4 ;(c) 6100-3 ;(d)$ [O 1$]-3$.

knot. The contours (logarithmic) are in units of $\sigma$; the total statistical dispersion of the measurement including photon and detector noise as well as the sky.

The main characteristics of the images are listed in Table 1. The FWHM of DD Tau as a whole ( $x$-direction) is given in column (4), while the FWHM of each one of the condensations along $y$ are given in columns (5) and (6), respectively. The separation (in arcseconds), P.A. (in degrees), and relative intensity of the two knots were estimated by center of gravity methods (e.g., Stobie 1980) and are given in columns (7), (8), and (9), respectively. Finally, the $\mathrm{S} / \mathrm{N}$ ratio of the peak is given in column (10). Variations in the spatial resolution are mainly due to the seeing. [ $\left.\mathrm{O}_{\mathrm{I}}\right]$ frames for $\mathrm{CZ}$ Tau were under exposed and are not listed.

\subsection{PSF and Continuum Images}

We find that $\mathrm{CZ}$ Tau is extended along the $y$-direction, (P.A. $=80^{\circ}$ ) and is therefore unsuitable for PSF determi- nations. We include the FWHM of CZ Tau in the $x$ - and $y$-directions (in cols. [2] and [3]) for completeness.

We used a faint star (located at 34" northwest of CZ Tau) in the continuum frame to determine the PSF. Its PSF has $\mathrm{FWHM}_{x}=0.64$, and FWHM $=0$. 62 and is shown in Figure 2. We obtained a best fit to the continuum image of DD Tau by co-adding two pointlike sources (two PSFs) whose separation is 0.55 , which are aligned in the direction P.A. $=6.4$, and for which DD Tau B is $10 \%$ brighter than DD Tau A. A leastsquares method was used to minimize the residuals in this fitting procedure. The residuals are, however, significant (residual contours at 2 and $3 \sigma$ ), and as seen in the last frame, they are concentrated in a small but extended area at the north end of DD Tau.

\subsection{Emission-Line Images}

The derived line flux values are summarized in Table 2 . The images were classified into two groups, according to their spatial resolution. The set $[\mathrm{S}$ II $]-1,[\mathrm{~N}$ II $]-4$, and $\mathrm{C} 6100-2$ have $\mathrm{FWHM}_{x} \simeq 1^{\prime \prime} .14 \pm 0.03$ and $\mathrm{FWHM}_{y} \simeq 0.75 \pm 0$ ".03, while the set $\left[\mathrm{O}_{\mathrm{I}}\right]-4,[\mathrm{~S} \mathrm{II}]-3,[\mathrm{~N}$ II $]-3$, and $\overline{\mathrm{C} 6} 100-3$ have $\mathrm{FWHM}_{x} \simeq 11^{\prime \prime} 09 \pm 0.03$ and $\mathrm{FWHM}_{y} \simeq 0$ 0.70 \pm 0.03 . The fluxes were integrated out to $10 \%$ of the peak intensity and normalized to the same exposure time (1200 s). The lowresolution spectrum of DD Tau published by Cohen \& Kuhi (1979) was used to estimate the continuum contribution in the lines region since the continuum filter is centered at $6100 \AA$. The line contribution to the total flux is given in column (3). The $[\mathrm{N}$ II $]-4, \mathrm{C} 6100-2$, [S II $]-3$, and C6100-3 images were taken in a sequence during the second night and the fluxes are far more reliable. The $15 \%$ higher flux in the first night's images is due to changes in the sky transparency between the two nights, since second night continua have been subtracted from images taken from the first night.

We find that $32 \%$ of the flux in the [N II] image comes from the $\left[\mathrm{N}_{\mathrm{II}}\right] \lambda 6584$ line, which agrees with the results of Edwards et al. (1989). A logarithmic contour plot of the net [N II] emission (continuum subtracted) is shown in Figure 3. This image shows that the [N II] emission is extended ("jetlike") in a manner that connects DD Tau B with DD Tau A. At much lower levels, the $[\mathrm{N}$ II $]$ emission appears to be extended in the direction P.A. $=130^{\circ}$.

We did not detect [O I] $\lambda 5577$ emission from DD Tau. This is to be expected since our filter is near the edge of the [O I]

TABLE 1

Data SUMmary

\begin{tabular}{|c|c|c|c|c|c|c|c|c|c|}
\hline \multirow[b]{2}{*}{ IMAGE } & \multicolumn{2}{|c|}{ CZ TAURI } & \multicolumn{7}{|c|}{ DD TAURI } \\
\hline & $\mathrm{FWHM}_{x}$ & FWHM $_{y}$ & FWHM $_{x}$ & FWHM $_{y, A}$ & FWHM $_{y, B}$ & Separation & P.A. & $I_{2} / I_{1}$ & $\mathrm{~S} / \mathrm{N}$ \\
\hline$\left[\begin{array}{lll}\mathrm{O} & 1\end{array}\right]-1$. & $\cdots$ & $\ldots$ & 1.04 & 0.63 & 0.67 & 0.46 & 0.4 & 1.30 & 01.04E2 \\
\hline$[\mathrm{O}$ I $]-2 \ldots \ldots \ldots$ & $\ldots$ & $\ldots$ & 1.06 & 0.67 & 0.69 & & & $\ldots$ & $01.80 \mathrm{E} 2$ \\
\hline$\left[\mathrm{O}_{1} \mathrm{I}\right]-3 \ldots \ldots \ldots$ & $\ldots$ & $\ldots$ & 1.06 & 0.68 & 0.68 & 0.42 & 2.8 & 1.13 & $01.72 \mathrm{E} 2$ \\
\hline$\left[\begin{array}{ll}\mathrm{O} & 1\end{array}\right]-4 \ldots \ldots \ldots$ & $\ldots$ & $\ldots$ & 1.02 & 0.70 & 0.73 & 0.46 & 7.2 & 1.11 & $00.83 \mathrm{E} 2$ \\
\hline$[\mathrm{S}$ III $]-1 \ldots \ldots \ldots$ & 0.74 & 0.79 & 1.14 & & & & & & $07.78 \mathrm{E} 2$ \\
\hline$[\mathrm{S}$ II $]-3 \ldots \ldots \ldots$ & 0.63 & 0.79 & 1.04 & 0.66 & 0.64 & 0.43 & 6.7 & 1.05 & $12.52 \mathrm{E} 2$ \\
\hline$\left[\begin{array}{l}\mathrm{S} \\
\mathrm{n}\end{array}\right]-4 \ldots \ldots \ldots$ & 0.54 & 0.69 & 1.09 & 0.57 & 0.55 & 0.50 & 5.5 & 1.03 & $15.81 \mathrm{E} 2$ \\
\hline$[\mathrm{N}$ II $]-1 \ldots \ldots \ldots$ & 0.53 & 0.75 & 1.04 & 0.68 & 0.68 & 0.48 & 8.2 & 1.14 & $14.28 \mathrm{E} 2$ \\
\hline$[\mathrm{N}$ II $]-2 \ldots \ldots \ldots$ & 0.53 & 0.69 & 1.02 & 0.66 & 0.63 & 0.47 & 6.6 & 1.25 & $14.92 \mathrm{E} 2$ \\
\hline$[\mathrm{N} n]-3 \ldots \ldots \ldots$ & 0.63 & 0.74 & 1.09 & 0.72 & 0.68 & & & $\ldots$ & $12.57 \mathrm{E} 2$ \\
\hline$[\mathrm{N} \mathrm{II}]-4 \ldots \ldots \ldots$ & 0.74 & 0.75 & 1.14 & 0.75 & 0.75 & 0.47 & 8.5 & 1.08 & $07.38 \mathrm{E} 2$ \\
\hline C6100-1 ........ & 0.77 & 0.88 & 1.21 & & & & & & $12.38 \mathrm{E} 2$ \\
\hline C6100-2 ........ & 0.67 & 0.82 & 1.14 & 0.73 & 0.73 & 0.36 & 7.2 & 1.08 & $27.09 \mathrm{E} 2$ \\
\hline C6100-3 ........ & 0.59 & 0.79 & 1.09 & 0.70 & 0.72 & 0.47 & $\ldots$ & 1.26 & $23.46 \mathrm{E} 2$ \\
\hline
\end{tabular}


No. 2, 1992

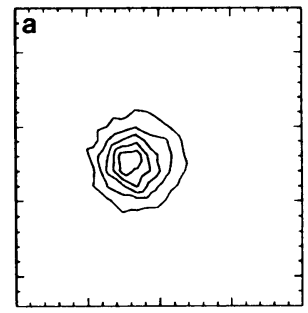

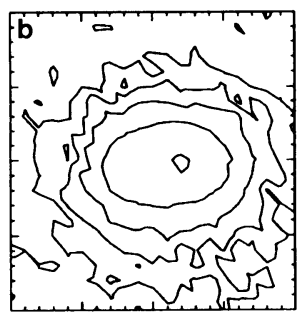
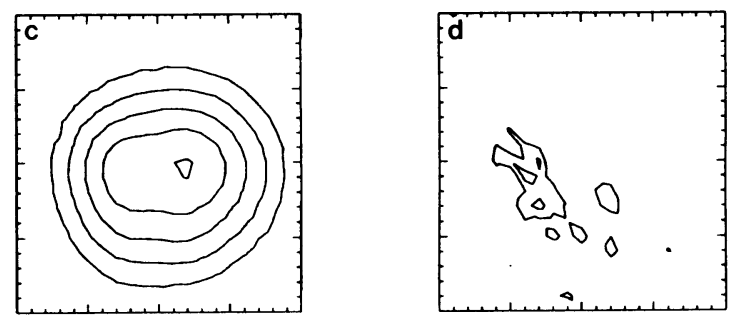

FIG. 2. - The left frame shows the PSF in the continuum C6100-3. The second frame shows the fit to C6100-3 image with two PSF separated by 0.55, oriented in the direction P.A. $=6.4$ and with relative intensity DD Tau $\mathrm{B} / \mathrm{DD}$ Tau $\mathrm{A}=1.1$. The third frame shows the original continuum (lowest contour, $16 \sigma$ ) image, and the last frame, the residuals of the best (least-squares) fit. (a) PSF; (b) fitted image; (c) $6100-3 ;(d)$ residuals.

25581 line. Indeed, the total flux is comparable to the continuum flux, once corrected for the $15 \%$ excess of the first night fluxes. The morphology of the $\left[\mathrm{O}_{\mathrm{I}}\right]$ images is similar to those in the continuum.

The flux levels in [S II] are very low. However, the morphology of the $[\mathrm{S} \mathrm{II}]$ image differs significantly from that of the continuum, indicating that there is indeed emission. We subtracted a continuum image from the [S II]-3 image (the former chosen to have similar seeing). The residuals are shown in Figure 3. Similar behavior is found when high-resolution images such as [S II]-4 and [O I]-3 (taken to be a highresolution continuum) are subtracted.

The continuum emission is wider and extends more to the west than the $[\mathrm{S} \mathrm{II}]$. This agrees with the results shown in Table 1, where the FWHM ${ }_{y, A}$ is smaller or equals $\mathrm{FWHM}_{y, B}$ in the continuum images ( $\mathrm{C}_{6} 100$ and [ $\left.\left.\mathrm{O}_{\mathrm{I}}\right]\right)$ and is larger than FWHM $_{y, B}$ in the $[\mathrm{N}$ II] and [S II] frames.

Summarizing, the FLER in DD Tau has been resolved on a scale of 0.5 in both [N II] and [S II]. The morphology of [N $\mathrm{II}]$ and [S II] emission is distinctly different from the continuum. In particular, the $[\mathrm{N}$ II] emission appears as a "jetlike" extension connecting the two continuum knots. The $[\mathrm{S} \mathrm{II}]$ emission seems to be confined to the northern knot.

\section{DISCUSSION}

\subsection{A Possible Binary?}

The two optical components we have described are coincident with two infrared sources detected in the $L$ band (Bouvier et al. 1991) using speckle techniques. The data are interpreted as a binary system of two TTSs, each surrounded with a small disk and a jet, and in which the jets are oriented E-W and are parallel with one another (Bouvier et al. 1992).

There are some difficulties with this model. It is not obvious how to reconcile the hypothesized jets in the E-W direction with the "jetlike" feature in our [N II] images that extends in

TABLE 2

FLUXES $^{\mathbf{a}}$

\begin{tabular}{ccc}
\hline \hline Image & Total Flux & $\begin{array}{c}\text { Percent Line } \\
\text { Flux } \\
(\%)\end{array}$ \\
\hline$[\mathrm{S} \mathrm{III}]-1 \ldots \ldots \ldots$ & $7.37 \times 10^{5}$ & 15 \\
{$[\mathrm{~N} \mathrm{II}]-4 \ldots \ldots \ldots$} & $4.76 \times 10^{5}$ & 32 \\
$\mathrm{C} 6100-2 \ldots \ldots \ldots$ & $2.93 \times 10^{6}$ & $\ldots$ \\
{$[\mathrm{O}$ I $]-4 \ldots \ldots \ldots$} & $4.94 \times 10^{4}$ & 13 \\
{$[\mathrm{~S} \mathrm{II}]-3 \ldots \ldots \ldots$} & $4.83 \times 10^{5}$ & 00 \\
{$[\mathrm{~N} \mathrm{II}]-3 \ldots \ldots \ldots$} & $3.71 \times 10^{5}$ & 42 \\
$\mathrm{C} 6100-3 \ldots \ldots \ldots$ & $2.07 \times 10^{6}$ & $\ldots$ \\
\hline
\end{tabular}

a The fluxes are given in instrumental units. the N-S direction and connects DD Tau A and DD Tau B. In order that both jets have blue- and redshifted components, Bouvier et al. argue that their disks are either optically thin, or small. This explanation is somewhat ad hoc since all other YSOs, some of which may be binary systems, show only blueshifted lines.

The P.A. of the infrared polarization vector in DD Tau is $125^{\circ}$, which is within $30^{\circ}$ of perpendicular to the axis connecting knots A and B. A nearly edge-on disk is the most natural explanation of this.

\subsection{Constraints on Theoretical Models}

Reasonable fits to some forbidden-line profiles were obtained in a model wherein a $200 \mathrm{~km} \mathrm{~s}^{-1}$ stellar wind impacts a surrounding flaring accretion disk (Hartmann \& Raymond 1988). This model predicts that emission arises in a diffuse region extending for 50-100 AU parallel to the disk plane. It is difficult to explain DD Tau with this model because the flow is already collimated on small scales $(\leq 50 \mathrm{AU})$ in a direction that is perpendicular to the expected disk plane.

Hydromagnetic winds from Keplerian accretion disks that achieve high enough (fast magnetosonic) Mach number $n \simeq$ $\left([4 \pi \rho]^{1 / 2} v_{\infty} / B\right.$ ) (where $\rho$ is the gas density, $B$ the magnetic field strength, and $v_{\infty}$ the terminal speed of the wind) can recollimate forming focal regions (Achterberg, Blandford, \& Goldreich 1983; Pelletier \& Pudritz 1992). The focal points are
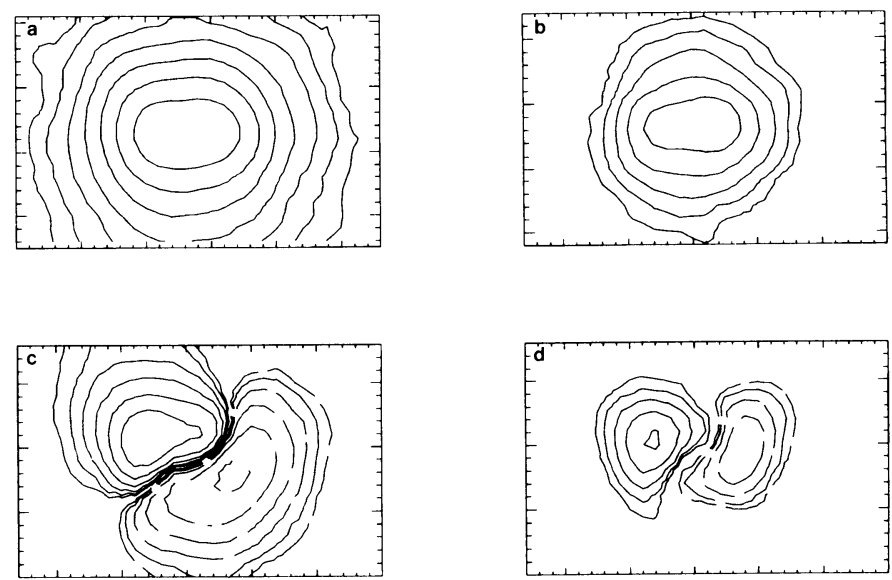

FIG. 3.-Contour plots of the [N II] emission are shown in $(a-b)$ frames; contour levels are $3,5,9,17,33,65,129$, and $257 \sigma$. [S II] emission are shown in the $(c-d)$; solid contours are the same as for [N II], dashed contours are the negative of these levels. $(a, c)$ Lower spatial resolution images after subtraction of the continuum frames scaled to the same total flux. (a) [N II]-C6100. (c) $[\mathrm{S} I I]-\mathrm{C} 6100$. On the right; higher resolution images where $\left[\mathrm{O}_{\mathrm{I}}\right]$ frames are

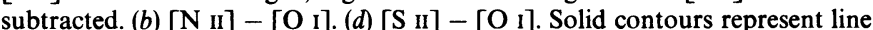
excess, and the dashed contours represent continuum excess. 
equidistant from the YSO above and below the disk plane. Mild MHD shocks occur at these positions whose excitation conditions and densities readily account for the FLERs (details in GP).

The data imply that the separation of the possible focal points and the source is $40 \mathrm{AU}$. The focal distance can be related to the maximum radius of the flow (GP; Pelletier \& Pudritz 1992), $\left(z_{\text {foc }} / r_{\max }\right)=2\left[3\left(n^{2}+1\right)\right]^{1 / 2}$. Taking $r_{\max } \simeq 2-4$ AU and $n \simeq 2.4$ for the critical Mach number for which flows begin to recollimate, we find $z_{\text {foc }} \simeq 20-40$ AU. Thus the separation and intensity of the knots $A$ and $B$ are consistent with the production of FLERs at focal points in our model. Broad-band emission is expected in the shocks since a large fraction of the energy released in disk accretion is carried by the disk wind and can be dumped into thermal energy at these shocks. Reprocessing of this emission by surrounding dust could account for the infrared images.

\subsection{Magnetic Focal Points?}

There are several challenges for a focal point interpretation of FLERs as well. If DD Tau is edge on, and the knots are focal points, then the central star must be hidden even at $L^{\prime}(3.87$ $\mu \mathrm{m})$. Using the scattering opacity of grain mixtures (e.g., Lazareff, Pudritz, \& Monin 1990), and the standard relation between the mean extinction and the column density $\bar{A}_{v}=$ $0.5\left(N / 10^{21} \mathrm{~cm}^{2}\right) \mathrm{mag}$, we find that the extinction required to hide a central source at $L^{\prime}$ is $\bar{A}_{v}=100 \mathrm{mag}$. We note that a star with an apparent $V$ magnitude less than 17.3 mag would not significantly contribute to the total flux in our frames.
It is puzzling that [S II] emission arises only from DD Tau A. This may imply that the system is slightly inclined so that DD Tau B is seen through more obscuring material. The fact that the IR polarization of $1.42 \%$ (Tamura \& Sato 1989) is not as high as might be expected for edge-on disks suggests a slight inclination of the system. The extension of knots A and B in a direction similar to the position angle of the IR polarization may arise from the scattering of light off circumstellar material.

In conclusion, we resolve the FLER of DD Tau into two sources which are somewhat extended and seemingly connected by $[\mathrm{N}$ II] emission. The data are consistent with the hypothesis that FLERs are shocks at focal points in recollimating, hydromagnetic disk winds. We cannot rule out the binary TTS model, however, without higher spatial resolution images.

We are indebted to Marshall McCall who loaned us his interference filters. An anonymous referee provided a very useful report. We thank our resident astronomer Jerome Bouvier and the staff at CFHT for their support during the observations, Domitilla de Martino and Daniel Ponz for helpful comments on data reduction, and Robert Lupton, Patricia Monger, and Douglas Welch for discussions. A. I. G. de C. thanks NSERC of Canada for an International Postdoctoral Fellowship, and R. E. P. thanks CITA for a Reinhardt Fellowship that allowed this work to be completed. This research was supported by NSERC of Canada.
Achterberg, B., Blandford, R. D., \& Goldreich, P. 1983, Nature, 304, 607 Appenzeller, I., \& Mundt, R. 1989, Astron. Ap. Rev., 3, 56

Bouvier, J., Tessier, E., \& Cabrit, S. 1992, A\&A, in press

Bouvier, J., Tessier, E., \& Perrier, C. 1991, CFH Inf Bull., 24, 15

Cabrit, S., Edwards, S., Strom, S. E., \& Strom, K. M. 1990, ApJ, 354, 687

Cohen, M., \& Kuhi, L. V. 1979, ApJS, 41, 743

Edwards, S., Cabrit, S., Ghandour, L. O., \& Strom, S. E. 1989, in Proc. ESO

Workshop on Low-Mass Star Formation, ed. B. Reipurth (Garching: ESO) 385

Edwards, S., Cabrit, S., Strom, S. E., Heyer, I., Strom, K. M., \& Anderson, E. 1987, ApJ, 321, 473

\section{REFERENCES}

Gomez de Castro, A. I., \& Pudritz, R. E. 1992, ApJ, 395, 501 (GP)

Hartmann, L., \& Raymond, J. C. 1988, ApJ, 337, 903

Lazareff, B., Pudritz, R. E., \& Monin, J-L. 1990, ApJ, 358, 170

McClure, R. D., et al. 1989, PASP, 101, 1156

Mundt, R., Ray, T. P., Buhrke, T., Raga, A. C., \& Solf, J. 1990, A\&A, 232, 37

Pelletier, G., \& Pudritz, R. E. 1992, ApJ, 394, 117

Stobie, R. S. 1980, Proc. SPIE, 264, 208

Tamura, M., \& Sato, S. 1989, AJ, 98, 1368 